\title{
Yeast Puf3 mutants reveal the complexity of Puf-RNA binding and identify a loop required for regulation of mRNA decay
}

\author{
S. SEAN HOUSHMANDI and WENDY M. OLIVAS \\ Department of Biology, University of Missouri-St. Louis, St. Louis, Missouri 63121-4499, USA
}

\begin{abstract}
The eukaryotic Puf proteins regulate mRNA translation and degradation by binding the $3^{\prime}$ untranslated regions of target mRNAs. Crystal structure analysis of a human Puf bound to RNA suggested a modular mode of binding, with specific amino acids within each of eight repeat domains contacting a single nucleotide of the target RNA. Here we study the mechanism by which the yeast Puf $3 p$ binds and stimulates the degradation of COX17 mRNA. Mutation of the predicted RNA-binding positions of Puf3p to those found in Puf5p demonstrated that a single amino acid change in Puf3p abolished detectable binding to COX17. Since this amino acid position in both Puf3p and Puf5p is predicted to contact an adenine in the respective target RNAs, the amino acid in Puf3p must play a more critical role in promoting $C O X 17$ interaction. In contrast, an amino acid change in the third repeat of Puf3p, which interacts with the only divergent nucleotide between the Puf3p and Puf5p targets, had no effect on binding COX17. These results argue that a simple set of rules cannot reliably link specific amino acid positions with target specificity. Each of these amino acid changes in Puf3p enhanced binding to the Puf5p target $H O$ RNA, suggesting a different mode of binding to this target. Finally, we identified an outer surface loop that was dispensable for binding but was required to promote both rapid deadenylation and subsequent decapping of the COX17 mRNA, most likely as a point of protein-protein interactions.
\end{abstract}

Keywords: Puf; decay; turnover; mRNA; yeast; 3'-UTR

\section{INTRODUCTION}

Regulation of mRNA-specific rates of translation and degradation is essential for proper control of gene expression. This type of regulation is especially apparent during early development, but it is also important in somatic cells and germline sex determination (Gray and Wickens 1998; Guhaniyogi and Brewer 2001). Such post-transcriptional control is commonly mediated by proteins that bind in a sequence-specific fashion to regulatory elements located in the $3^{\prime}$ untranslated regions (UTRs) of mRNAs (Derrigo et al. 2000; Grzybowska et al. 2001; Mazumder et al. 2003). However, the mechanisms by which these proteins either promote or inhibit translation and/or degradation of the bound mRNAs are poorly understood.

The Puf family of proteins is one group of $3^{\prime}$-UTRbinding proteins that regulate both translation and mRNA

Reprint requests to: Wendy M. Olivas, Department of Biology, University of Missouri-St. Louis, One University Boulevard, St. Louis, MO 63121-4499, USA. e-mail: olivasw@umsl.edu; fax: (314) 516-6233.

Article and publication are at http://www.rnajournal.org/cgi/doi/10.1261/ rna.2168505. degradation in diverse eukaryotic organisms (Wickens et al. 2002). Pumilio from Drosophila melanogaster (DmPum) and $\mathrm{FBF}$ from Caenorhabditis elegans were the founding members of this group, thus providing the Puf family name. Both DmPum and FBF require interactions with other proteins to regulate mRNA expression. For example, DmPum must form a complex with Nanos and Brat proteins to regulate hunchback mRNA (Sonada and Wharton 1999, 2001), whereas Nanos but not Brat is recruited for regulation of cyclin B mRNA (Sonada and Wharton 2001). Similarly, FBF interacts with a Nanos-like protein to regulate fem-3 mRNA (Kraemer et al. 1999), as well as a CPEB (cytoplasmic polyadenylation element-binding protein) homolog for a possible role in spermatogenesis (Luitjens et al. 2000). While the requirement for protein partners is likely true of all Puf-mediated mRNA regulation, Puf partners have yet to be identified in unicellular eukaryotes.

Puf proteins are characterized by a domain containing eight imperfect repeats of a 36 -amino-acid sequence plus short flanking regions. This Puf repeat domain is sufficient not only for mRNA binding (Zamore et al. 1997; Zhang et al. 1997; Jackson et al. 2004) but also for interacting with protein partners (Kraemer et al. 1999; Sonada and Wharton 
1999, 2001; Luitjens et al. 2000) and, at least in DmPum and the yeast Puf3p, for regulating mRNA metabolism (Wharton et al. 1998; Jackson et al. 2004). The crystal structures of the repeat domains of DmPum (Edwards et al. 2001) and a human Puf protein (HsPum) (Wang et al. 2001) are similar. In both, each repeat folds into three $\alpha$ helices that stack on the helices of neighboring repeats to form an extended crescent-shaped structure. The core consensus sequences of each repeat are arranged on parallel helices located on the inner concave surface. The crystal structure of HsPum bound to an RNA ligand confirmed that this inner surface binds RNA, and the binding was predicted to be modular, with three amino acids of each repeat recognizing a successive base along the RNA (Wang et al. 2002). Conversely, mutational analysis of DmPum indicates that amino acids on the outer convex surface contact Nanos and Brat proteins (Edwards et al. 2001).

The binding sequences of all RNA targets analyzed to date contain a shared UGUR motif required for Puf binding, with flanking sequences providing specificity (Wickens et al. 2002). Inspection of the RNA target sequences of DmPum and its most closely related Puf proteins, including yeast Puf $3 p$ and Pufs in human, mouse, and Xenopus, reveals an expanded, shared binding motif of UGUANAUA (Murata and Wharton 1995; Zamore et al. 1997; Nakahata et al. 2001; White et al. 2001; Jackson et al. 2004). The crystal structure of HsPum shows that nucleotides (nt) 1 through 8 of this RNA motif are contacted by protein repeats 8 through 1 , respectively (Wang et al. 2002).

Saccharomyces cerevisiae contains six members of the Puf protein family (Puflp-Puf6p). To date, only three of the yeast Pufs have verified roles in regulating specific RNA targets. Puf3p binds the $3^{\prime}$-UTR of COX17 mRNA and promotes its deadenylation and subsequent decay (Olivas and Parker 2000), Puf5p binds the $3^{\prime}$-UTR of the $H O$ mRNA, repressing its expression and stimulating its decay (Tadauchi et al. 2001), and Puf6p binds the $3^{\prime}$-UTR of the ASH1 mRNA to regulate its translation and localization (Gu et al. 2004). In addition to these studied targets, a microarray analysis has identified several hundred candidate RNA targets that interact with one or more of the yeast Pufs 1-5 (Gerber et al. 2004). Moreover, consensus sequence motifs containing UGUR were identified in many of the RNAs associated with Pufs 3, 4, and 5, with RNAs bound by each Puf protein having distinct sequences following the UGUR. However, it is still unclear how each of the yeast Pufs recognizes its unique target RNA sequence, or how the bound Pufs promote functional changes of the mRNAs.

In this work we have focused on understanding how yeast Puf3p attains specificity to its mRNA target. The predicted RNA-binding residues in Puf3p and Puf5p are identical in all but three repeats. By mutating those residues of Puf3p to those found in Puf5p, we showed that a single amino acid change was sufficient to prevent detectable binding of the protein to its COX17 mRNA target, whereas replacing other residues had no effect on binding. Conversely, any combination of these amino acid changes in Puf3p enhanced binding of the protein to the Puf5p target HO mRNA, with each amino acid playing a small but similar role in binding affinity. These results suggest that corresponding amino acid positions in Puf3p and Puf5p have divergent roles in determining target specificity, and therefore, the mode of binding of these Pufs to their target RNAs is likely different. We also identified a loop region on the outer surface of Puf3p that was required for promoting both deadenylation and subsequent decapping of the COX17 mRNA, indicating that regulation of these processes is linked by a single interaction point on Puf3p.

\section{RESULTS}

\section{Creation of Puf3RDp mutants based on sequence and structural alignments}

The repeat domain of the Puf3 protein (Puf3RDp) is sufficient not only for in vitro binding to COX17 mRNA but also for in vivo regulation of this transcript's decay (Jackson et al. 2004). Here we characterized the specific amino acids of the Puf3RDp involved in binding and regulation of COX17 mRNA. The crystal structure of HsPum bound to RNA shows that each base is recognized by amino acids located at three conserved positions within an individual Puf repeat domain (Wang et al. 2002). An alignment of the repeat domains of HsPum, DmPum, and Puf3p reveals that the amino acids of HsPum that interact with RNA bases are absolutely conserved with those of DmPum and Puf3p (Wang et al. 2002). Further support that these conserved positions within Puf3p make specific RNA interactions is that the optimal RNA target sequence of Puf3p is identical to that of the NRE1 boxB bound by DmPum and to the sequence bound by HsPum in crystal structures (Zamore et al. 1999; Wang et al. 2002; Jackson et al. 2004).

To determine which amino acids of Puf3p promoted specificity of binding to the COX17 mRNA, we analyzed a sequence alignment (Wang et al. 2002) of Puf3p with another yeast Puf protein, Puf5p (Fig. 1A). A comparison of the amino acids located at the predicted RNA-interacting positions of Puf3p and Puf5p revealed only three differences between the two proteins, one each in repeats 1,3 , and 5 (boxed in green, olive, and magenta, respectively, Fig. 1A). The likely Puf5p target region within the HO $3^{\prime}$-UTR is very similar to the Puf3p target (Fig. 1D), yet Puf5p cannot bind the COX17 target sequence (Jackson et al. 2004), and Puf3p binds only weakly to the HO target sequence (see below). This made the amino acid difference in repeat 3 especially intriguing, since in the HsPum-RNA complex, the third repeat interacts with the sixth nucleotide position of the RNA sequence (UGUAUAUA). This position is the only difference between the Puf3p target sequence and the 
(A)

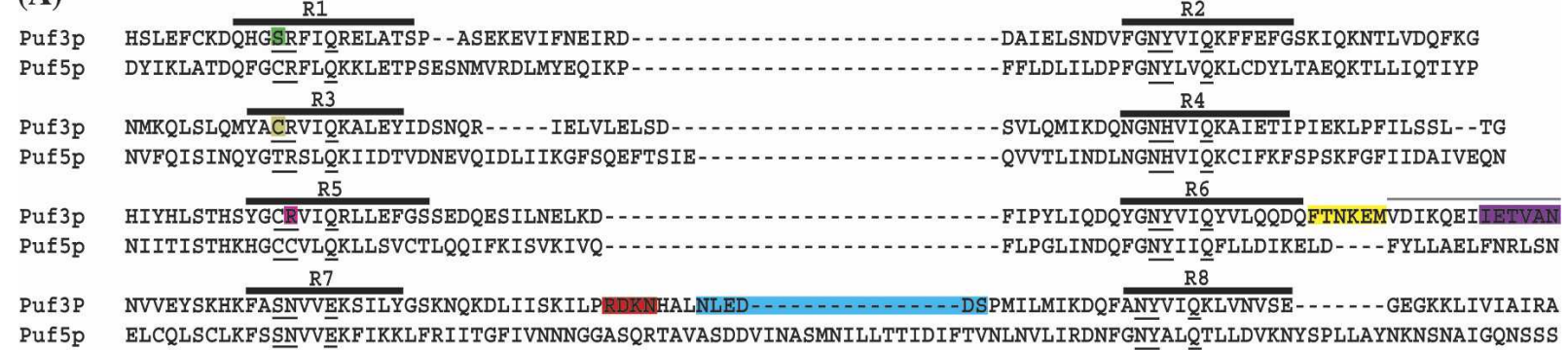

(B)

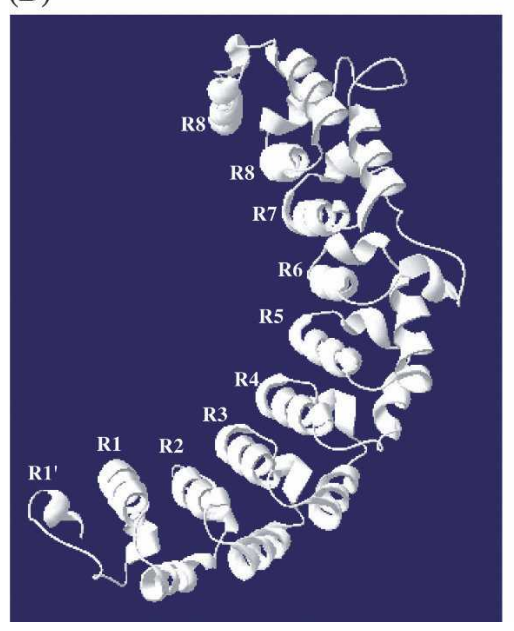

(C)

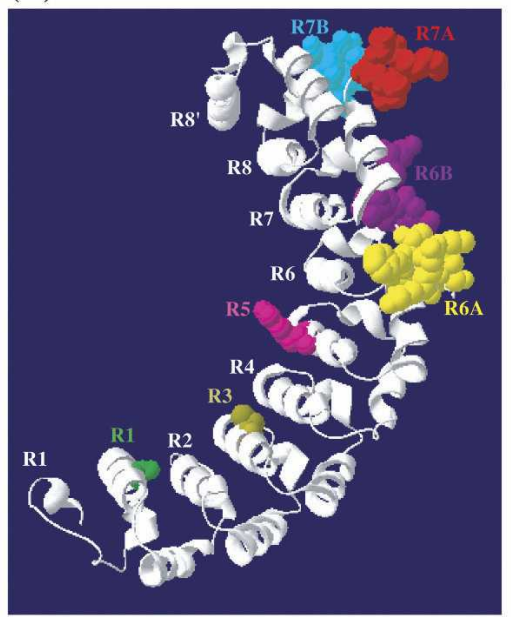

(D)

\begin{tabular}{ll} 
Puf Repeat: & 87654321 \\
\hline Puf $3 p /$ COX17 & UGUAUAUA \\
Puf5p/HO & UGUAUGUA
\end{tabular}

Puf $5 \mathrm{p} /$ HO

FIGURE 1. The Puf3p Repeat Domain. (A) Amino acid sequence alignment of the Puf3p and Puf5p Repeat Domains (Wang et al. 2002). Each repeat is numbered R1-R8, with thick lines above each repeat indicating the predicted regions of the RNA-interacting helices. Individual amino acids predicted to make RNA contacts are underlined. The region predicted to form the third helix of repeat 6 is marked over the sequence with a thin line. Amino acids mutated in this study are as follows: Amino acids predicted to make van der Waals interactions with the RNA are in green (R1) and olive (R3) boxes, and an amino acid predicted to make a stacking interaction with the RNA is in a magenta (R5) box. Regions of amino acids deleted in this study are as follows: Regions predicted to be positioned in an outer surface loop within repeat 6 are in yellow (R6A) and violet (R6B) boxes, and regions predicted to be positioned in an outer surface loop following repeat 7 are in red (R7A) and azure (R7B) boxes. (B) Predicted Puf3RDp structure created by Swiss-Model (37-39). (C) The locations of all mutated and deleted amino acids on the Puf3RDp structure are indicated with colors corresponding to the respective boxes in panel $A$. (D) Alignment of target RNA sequences from the COX17 and $H O$ mRNAs bound by the Puf3p and Puf5p proteins, respectively. The HO sequence is derived from our yeast strains and is identical to that found in the Saccharomyces Genome Database, although it differs by $1 \mathrm{nt}$ from the sequence listed by Tadauchi et al. (2001) (UGUGUGUA). The Puf repeat predicted to interact with each nucleotide is indicated above the sequences (Wang et al. 2002).

predicted Puf5p target sequence (Fig. 1D). We therefore exchanged the three differential amino acids of Puf3p with the respective amino acids of Puf5p. Combinations of these point mutations were also made to test whether multiple amino acid changes were necessary to alter binding specificity. A prediction of where these amino acid positions might be located on a theoretical structure of Puf3RDp is shown in Figure 1C. This structure was created by SwissModel (Peitsch 1995; Guex and Peitsch 1997; Schwede et al. 2003) by aligning Puf3RDp with Puf proteins of known structure.

In addition to testing Puf3RDp interactions involved in RNA binding specificity, we wished to characterize Puf3RDp amino acids involved in regulation of COX17 mRNA decay. In the case of DmPum, interactions with Nanos and Brat map to loop structures on the outer convex surface of DmPum between repeats 6, 7, and 8 (Edwards et al. 2001). Analysis of our modeled Puf3RDp structure shows two outer surface loops in the same regions between repeats 6, 7, and 8 (Fig. 1B). The extended loop located between repeats 7 and 8 encompasses 16 amino acids, 11 amino acids larger than the equivalent loop in DmPum. To analyze whether any part of this loop is involved in RNA decay regulation, two different deletion mutants were made encompassing amino acids 3-6 or 10-15 of the loop (Fig. 1). The outer surface loop between repeats 6 and 7 is more ambiguous in terms of structure. A sequence alignment of Puf3RDp with HsPum and DmPum (Wang et al. 2002) showed that Puf3RDp contains an extra six amino acids located between the latter two $\alpha$-helix domains of repeat 6 (Fig. 1A, boxed in yellow). These six amino acids could form a surface loop unique to the Puf3 protein that might be involved in mRNA decay regulation. In contrast, the Swiss-Model prediction of the Puf3RDp structure places 
these six amino acids into the third helix of repeat 6 (compare the location of R6A in Fig. 1B,C), while placing the last six amino acids of repeat 6 into a loop between repeats 6 and 7 (R6B in Fig. 1B). To test these predictions, deletion mutants were made of these two regions.

\section{Analysis of Puf3RDp sequences required for COX17 mRNA interaction}

To test whether any of the mutations or deletions in Puf3RDp affect the protein's ability to bind the COX17 $3^{\prime}$ UTR target sequence, in vitro binding assays were performed with glutathione-S-transferase (GST)-tagged wildtype and mutant proteins. The RNA used in these assays encompassed the COX17 Site A sequence (Fig. 2A), which we previously found to be the higher affinity target of two Puf3p binding sequences in the COX17 3'-UTR (Jackson et al. 2004). The resulting complexes were analyzed by gel mobility shift experiments. As shown in Figure 2B, wildtype Puf3RDp bound to the Site A RNA (lane 3), while GST protein alone did not bind (lane 2). Point mutations in R3 (lane 7), R5 (lane 9), or both R3/R5 (lane 8) did not disrupt binding. In contrast, a point mutation in R1 (lane 4), or any double mutation with R1 (R1/R3 in lane 5 or R1/R5 in lane 6), inhibited detectable RNA binding. This indicates that of these three amino acid differences between Puf3p and Puf5p, only the S553C change in the R1 mutant alters binding to the Puf3p target.

(A)

(B)

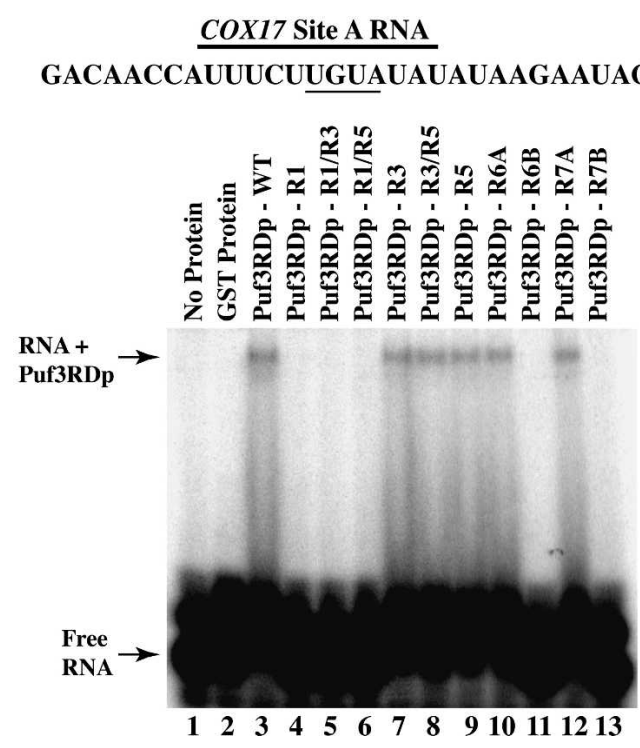

Deletions in the predicted outer surface loops of Puf3RDp should not affect RNA binding to the inner surface of the protein unless a deletion alters the overall structure of the protein. As expected, the R6A and the R7A deletions (Fig. 2B, lanes 10,12) did not disrupt RNA binding. However, the R6B and R7B deletions (Fig. 2B, lanes 11,13) inhibited detectable binding, most likely due to altered protein structure. The results of the R6A and R6B deletion mutants support the sequence alignment of these amino acids in Figure 1A versus the Swiss-Model structural prediction of these amino acids in Figure 1B. Specifically, the R6A region that is dispensable for binding is more likely to be in an outer surface loop, while the $\mathrm{R} 6 \mathrm{~B}$ region that is required for binding is more likely to be in the structurally important third helix of repeat 6 .

Gel mobility shift assays were also performed in the presence of excess nonspecific or specific unlabeled competitor RNA (Fig. 2C). All interactions of the wild-type and binding-competent mutant Puf3RD proteins with the Site A RNA were specific. Excess nonspecific vector RNA had no effect on binding, while excess COX17 3'-UTR RNA abolished the signal from the bound complex (Fig. 2C). While only a small amount of specific complex is seen in these RNA binding assays, we believe this may be due to decay of the complexes during the gel run as evidenced by the significant smearing of RNA signal down each lane of bound RNA. Moreover, all signal through these lanes is abolished by specific competitor (Fig. 2C), providing further evidence that the smeared signal represents specific complexes.

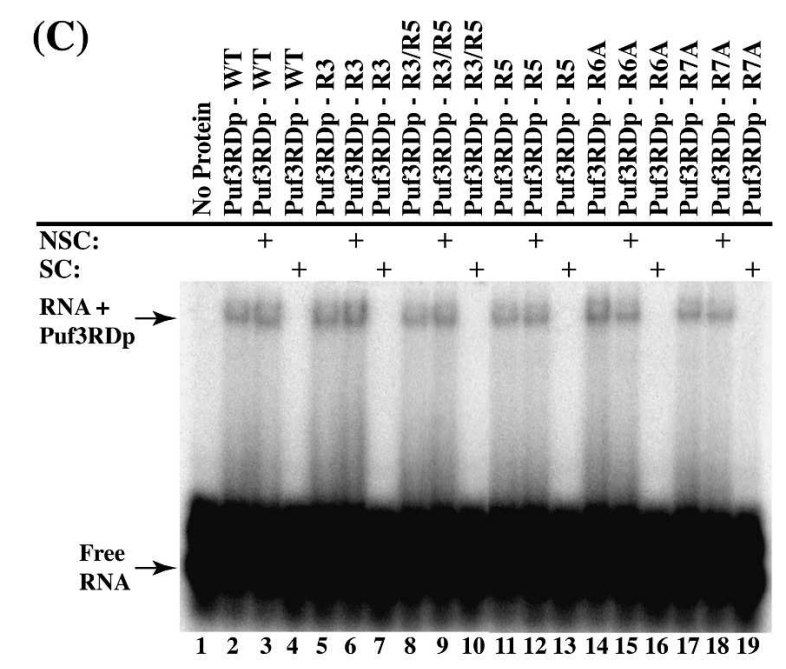

FIGURE 2. In vitro binding of wild-type and mutant Puf3RD proteins to COX17 Site A RNA. (A) Sequence of the 30-nt COX17 Site A transcript used in binding reactions is shown. The UGUA core binding element is underlined. $(B, C)$ In vitro binding reactions of radiolabeled COX17 Site A RNA in the absence or presence of $0.65 \mu \mathrm{M}$ protein were separated on native polyacrylamide gels. Positions of unbound RNA (Free RNA) as well as RNA-Puf3RDp complexes (RNA+Puf3RDp) are indicated. (B) Reactions were performed in the presence of GST alone (lane 2) wild-type Puf3RDp (lane 3), or mutant Puf3RD proteins (lanes 4-13). (C) The specificity of RNA-protein interactions was analyzed by using excess unlabeled vector RNA or full-length COX17 3'-UTR RNA as nonspecific (NSC) or specific (SC) competitors, respectively, in binding reactions with wild-type Puf3RDp (lanes 2-4) or mutant Puf3RD proteins (lanes 5-19). The presence of either competitor is marked (+). 
To test whether the binding-competent mutant proteins altered RNA binding affinity, the apparent equilibrium dissociation constant $\left(K_{\mathrm{d} \text {,app }}\right)$ was measured for each protein. Figure 3A shows representative gel mobility shift assays, while the binding data from all gel mobility shift assays are plotted in Figure 3B. Complex formation was measured as the sum of all specific complexes migrating through each lane. Binding-incompetent RNAs appeared in some RNA preparations with a slightly retarded mobility versus Free RNA, and these RNA bands were not included in the calculations of Free RNA or bound RNA (see Materials and Methods). We found no significant differences in the affinities of the binding-competent mutant proteins to that of the wild-type protein $\left(K_{\mathrm{d} \text {,app }}=0.50 \pm 0.02 \mu \mathrm{M}\right)$ (Fig. 3B). We have previously shown the specific activity of our wild-type Puf3RDp to be at 5\%-10\% of total protein concentration (Jackson et al. 2004). Therefore, we estimate that our apparent $K_{\mathrm{d}}$ is at least 10-fold weaker than the actual $K_{\mathrm{d}}$. Since all proteins were purified in the same manner, the specific activity of all mutant proteins is predicted to be similar to that of the wild-type Puf3RDp. This prediction is supported by the fact that all proteins tested in Figure $3 \mathrm{~B}$ displayed the same $K_{\mathrm{d} \text {,app }}$ value. Together, these results indicate that unlike the $\mathrm{R} 1$ mutation, the amino acid differences in R3 and R5 between Puf3p and Puf5p play no role in the differential binding affinities of the two proteins toward the Puf3p target RNA. Moreover, the amino acids of the $\mathrm{R} 6 \mathrm{~A}$ and $\mathrm{R} 7 \mathrm{~A}$ regions that are likely located in outer surface loop structures have no role in Puf3RDp RNA binding affinity.

\section{An outer surface loop of the Puf3RDp is required for RNA decay regulation}

Amino acids in the outer surface loops are prime candidates for interacting with other proteins involved in mRNA decay. Therefore, in vivo transcriptional shut-off assays were performed to test for the functional rescue of a yeast puf $3 \Delta$ strain via each mutant protein by monitoring the decay of steady-state COX17 mRNA. For this experiment, plasmids encoding the wild-type or mutant proteins were transformed into a $p u f 3 \Delta$ strain containing a temperaturesensitive lesion in RNA polymerase II ( $r p b 1-1)$, in which transcription is rapidly repressed following a shift to the nonpermissive temperature.

In the puf $3 \Delta$ strain, COX17 mRNA decayed with a halflife of $28.0 \pm 0.7 \mathrm{~min}$, while expression of the wild-type Puf3RDp in the $p u f 3 \Delta$ strain rescued rapid decay of COX17 mRNA to a half-life of $15.5 \pm 0.7 \mathrm{~min}$ (Fig. $4 \mathrm{~A}, \mathrm{~B}$ ). When mutant proteins that could not bind the COX17 mRNA (Puf3RDp-R1, -R1/R3, -R1/R5, -R6B, and -R7B) were expressed in the $p u f 3 \Delta$ strain, COX17 decayed with a halflife of 28-30 min, nearly identical to that of the puf $3 \Delta$ alone (Fig. 4C-E,J,L). This indicates that RNA binding is required for Puf3RDp-mediated rapid COX17 mRNA decay. Conversely, when proteins containing inner surface point muta-

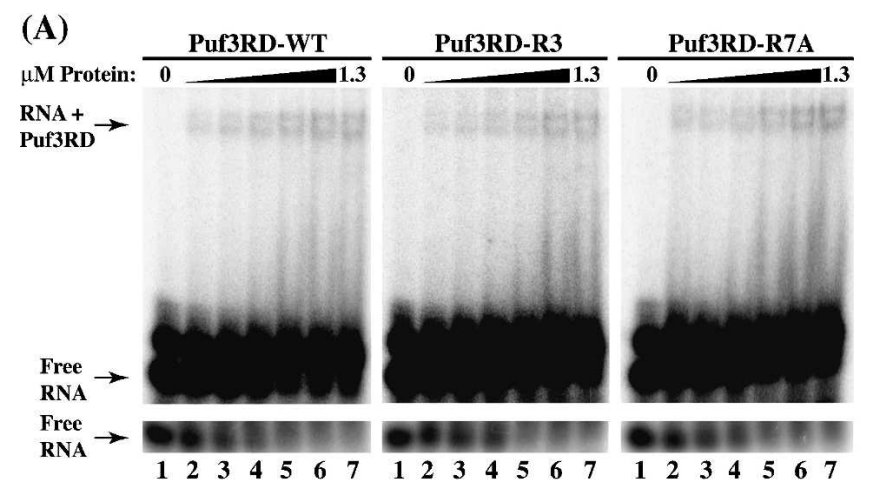

(B)

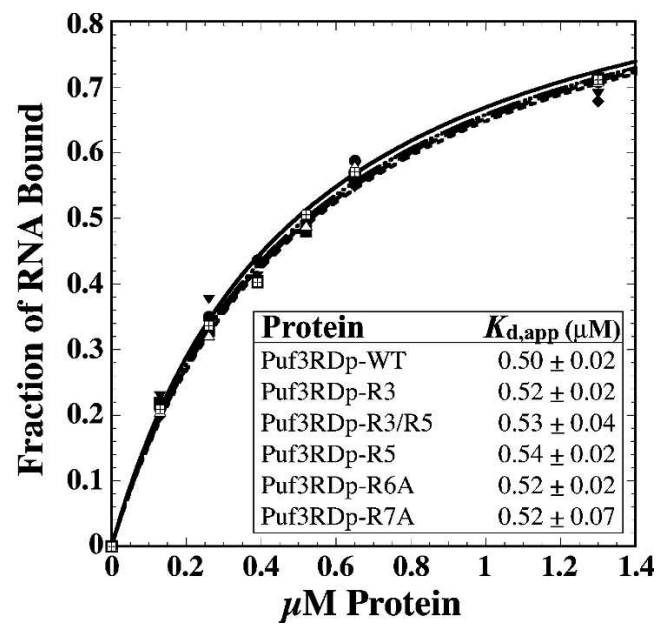

FIGURE 3. Comparison of binding affinities of wild-type and mutant Puf3RD proteins to COX17 Site A RNA in vitro. (A) Gel mobility shift assays of in vitro binding reactions of radiolabeled COX17 Site A RNA in the absence or presence of increasing concentrations of Puf3RDp-WT, Puf3RDp-R3, and Puf3RDp-R7A are shown as representatives of similar assays performed on all mutant proteins. Concentrations of protein used in binding reactions were $0,0.13,0.26,0.39,0.52,0.65$, and $1.3 \mu \mathrm{M}$ in lanes $1-7$, respectively. Positions of unbound RNA (Free RNA) as well as RNA-Puf3RDp complexes (RNA+Puf3RDp) are indicated. Panels below each gel reflect a lighter exposure of the Free RNA within each respective gel. (B) Data from the gel mobility shift assays performed with Puf3RDp-WT and all binding-competent mutant Puf3RD proteins are plotted with the micromolar concentration of protein used in the binding reaction on the $X$-axis and fraction of RNA shifted from free RNA to bound RNA on the $Y$-axis. Binding curves are shown for Puf3RDp-WT (filled circle), Puf3RDp-R3 (filled inverted triangle), Puf3RDp-R3/R5 (filled square), Puf3RDp-R5 (filled diamond), Puf3RDp-R6A (open triangle), and Puf3RDp-R7A (open crossed square). Data points are averages of multiple experiments. The $K_{\mathrm{d} \text {,app }}$ values calculated for each protein are listed. 
$\begin{array}{llllllllllll}\text { Min: } & 0 & 2 & 4 & 6 & 8 & 10 & 15 & 20 & 30 & 60 & t_{1 / 2}\end{array}$

(A) $p u f 3 \Delta$

(B) $p u f 3 \Delta+p P u f 3 R D-W T$

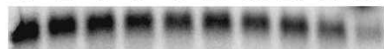

$28.0 \pm 0.7$

$15.5 \pm 0.7$

(C) $p u f 3 \Delta+p P u f 3 R D-R$

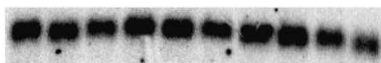

$28.5 \pm 0.7$

(D) $p u f 3 \Delta+p P u f 3 R D-R 1 / R 3$

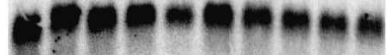

$29.8 \pm 0.4$

(E) $p u f 3 \Delta+p P u f 3 R D-R 1 / R 5$

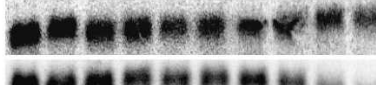

(F) $p u f 3 \Delta+p P u f 3 R D-R 3$

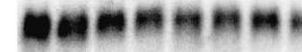

$29.3 \pm 0.4$

$16.3 \pm 0.4$

(G) $p u f 3 \Delta+p P u f 3 R D-R 3 / R 5$

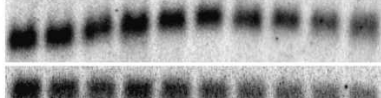

(H) $p u f 3 \Delta+p P u f 3 R D-R 5$

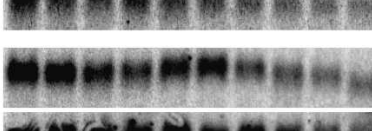

(I) $p u f 3 \Delta+p P u f 3 R D-R 6 A$

(J) $p u f 3 \Delta+p P u f 3 R D-R 6 B$

(K) $p u f 3 \Delta+p P u f 3 R D-R 7 A$

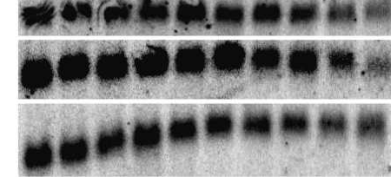

$16.8 \pm 0.4$

$16.5 \pm 0.4$

$16.8 \pm 0.4$

$30.0 \pm 1.4$

$29.0 \pm 0.7$

$30.5 \pm 1.4$

FIGURE 4. COX17 mRNA decay rates in the presence of wild-type or mutant Puf3RD proteins in vivo. Shown are Northern blot analyses of the decay of COX17 mRNA from a puf $3 \Delta$ strain transformed with or without constructs expressing either Puf3RDp-WT or each of the mutant Puf3RD proteins. Minutes following transcriptional repression are indicated above the sets of blots, with the half-lives $\pm \mathrm{SD}\left(\mathrm{t}_{1 / 2}\right)$ as determined from multiple experiments.

tions that did not inhibit RNA binding (Puf3RDp-R3, -R3/R5, and -R5) were expressed in the puf3 $\Delta$ strain, COX17 decayed with a half-life of $16-17 \mathrm{~min}$, nearly identical to wild-type Puf3RDp (Fig. 4F-H). This demonstrates that these mutations have no detrimental effect on mRNA decay regulation. Most interesting were our results with the outer surface loop deletions that did not disrupt RNA binding. Expression of Puf3RDp-R6A in the puf3s strain rescued COX17 mRNA decay to wild-type levels, with a half-life of $16.8 \pm 0.4 \mathrm{~min}$ (Fig. 4I). Thus, this outer loop region plays no role in signaling for the decay of COX17 mRNA. However, expression of Puf3RDp-R7A in the $p u f 3 \Delta$ strain did not rescue rapid decay of COX17 mRNA, with a half-life of $29.0 \pm 0.7 \mathrm{~min}$ (Fig. $4 \mathrm{~K}$ ). Therefore, the amino acids of the R7A loop are absolutely essential for Puf3RDp to mediate rapid mRNA decay. We predict that this region may be involved in protein-protein interactions that signal to the decay machinery.

\section{The regulation of COX17 mRNA by the mutant Puf3RD proteins is mediated through deadenylation and decapping}

Puf3p regulates COX17 mRNA decay by promoting both rapid deadenylation and rapid decapping (Olivas and Parker 2000); therefore, the above differences in the halflife of COX17 mRNA could be due to differences in the rate of deadenylation, decapping, or both. To examine these steps of decay, in vivo transcriptional pulse-chase assays were performed. For these experiments, a pulse of newly synthesized transcripts was created whose deadenylation and subsequent decay could be monitored over time.

In confirmation of previous work (Jackson et al. 2004), we found that a pulse of COX17 transcripts expressed in a puf3 $3 \Delta$ strain was synthesized with poly(A) tails of 35-60 residues (Fig. 5A, lane 0 ). The poly $(\mathrm{A})$ tails then deadenylated slowly such that fully deadenylated species did not appear until 15 min after transcriptional repression, and transcripts with short poly(A) tails of between 0 and 25 residues persisted to $40 \mathrm{~min}$ (Fig. 5A). In contrast, expression of the wild-type Puf3RDp in the puf $3 \Delta$ strain rescued rapid deadenylation, with transcripts that started out with similar poly(A) tails of 45-60 residues (Fig. 5B, lane 0) reaching a deadenylated state within $4 \mathrm{~min}$. Moreover, there was no persistence of transcripts with short poly(A) tails, and all transcripts were nearly completely degraded by 6 min (Fig. 5B). This indicates that Puf3RDp is sufficient not only to promote rapid deadenylation but also to promote a second step in COX17 degradation. Since terminal deadenylation is not typically required for subsequent decapping (Caponigro and Parker 1996), the simplest explanation is that Puf3RDp accelerates decapping of deadenylated and partially deadenylated COX17 mRNAs (Olivas and Parker 2000).

The expression of each mutant Puf3RD protein in the puf $3 \Delta$ strain was analyzed for the ability to promote rapid COX17 deadenylation and decapping. As predicted, any mutant protein that failed to bind COX17 mRNA also failed to promote rapid deadenylation and decapping. Figure 5C shows a representative Northern blot analysis for such a mutant protein, Puf3RDp-R1. The slow deadenylation and persistence of transcripts with short poly(A) tails in this and other binding-incompetent mutants looked identical to that seen in the puf3 $\Delta$ strain (Fig. 5, cf. $\mathrm{C}$ and $\mathrm{A}$ ). For the mutant proteins that could bind COX17 mRNA, all but one promoted rapid deadenylation and decapping. A representative Northern blot analysis of one such protein, Puf3RDp-R3/R5, is shown in Figure 5D. Deadenylation proceeded rapidly in the binding-competent mutants, with no persistence of transcripts with short poly(A) tails and nearly complete degradation by 6 min. This is identical to the pattern seen with the wild-type Puf3RDp (Fig. 5, cf. D and B). In contrast, the Puf3RDpR7A mutant failed to promote rapid deadenylation and decapping (Fig. 5E). Transcripts did not reach a deadenylated state until $15 \mathrm{~min}$ and then persisted with short poly(A) tails to $40 \mathrm{~min}$, a pattern identical to that seen in the puf $3 \Delta$ strain (Fig. 5, cf. E and A). This result suggests that while the R7A loop region has no role in binding to the COX17 mRNA, it is absolutely required for interactions that mediate signals to both the deadenylation and decapping machinery. 
(A)

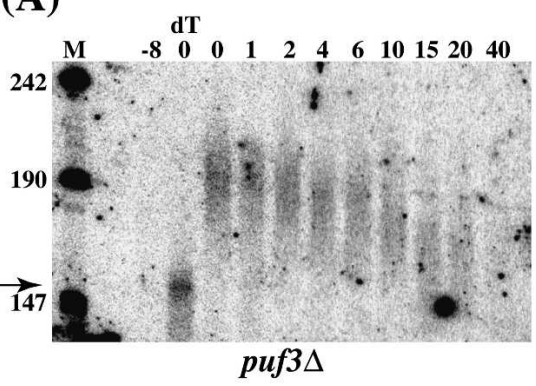

(C)

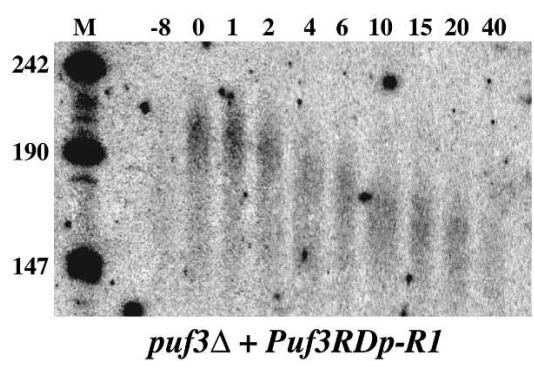

(E)

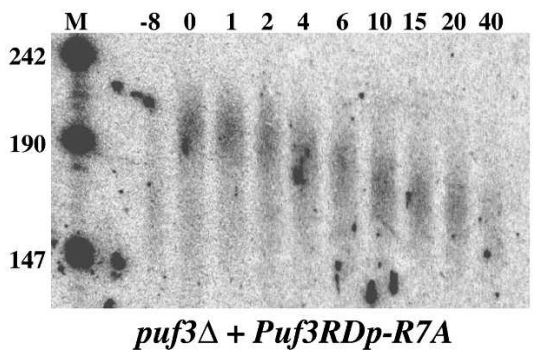

(B)

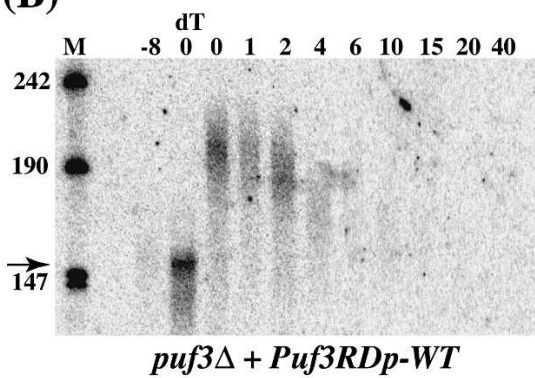

(D)

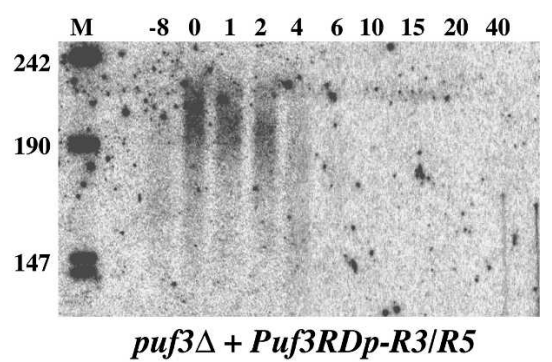

formation with each of the mutant proteins showed that all single and double point mutations on the RNA binding surface of Puf3RDp maintained binding to the HO RNA (lanes 5-10). In addition, the R6A and R7A outer surface deletions that did not inhibit binding to the COX17 RNA also did not inhibit binding to the HO RNA (lanes 11,13, respectively). In contrast, the R6B and R7B outer surface deletions prevented detectable binding of the protein to the HO RNA (lanes 12,14, respectively). Since these deletions also prevented binding to the COX17 RNA, these results provide additional evidence that the R6B and R7B deletions cause overall structural changes to the Puf3RDp that block its ability to bind RNA.

To verify that all of the complexes formed with the HO RNA were sequence specific, each protein was incubated with the radiolabeled $H O$ target RNA in the presence or absence of excess unlabeled $H O$ target RNA as a specific competitor or unlabeled vector RNA as a nonspecific competitor. As shown in Figure 6B, all interactions were specific since they could be competed with excess specific, but not nonspecific, competitor.

Finally, to determine whether any of the mutant proteins bound the HO RNA with an increased affinity versus the wild-type Puf3RDp, the $K_{\mathrm{d} \text {,app }}$ was measured for each protein. Three representative gel mobility shift assays are shown in Figure $7 \mathrm{~A}$, while the data from all gel mobility shift analyses are plotted in the graph of Figure 7B. For the wild-type Puf5RDp, the $K_{\text {d,app }}$ was $0.27 \pm 0.02 \mu \mathrm{M}$ (Fig. $\left.7 \mathrm{~A}, \mathrm{~B}\right)$. In contrast, the interaction of Puf3RDp with $H O$ RNA was approximately fivefold

\section{Point mutations in Puf3RDp enhance affinity to a Puf $5 p$ target mRNA}

Since each of the three inner surface mutations replaced an amino acid of Puf3RDp with the respective amino acid found in Puf5p, we hypothesized that these mutations might enhance binding to the $H O$ mRNA target. To examine RNA-protein complex formation, gel mobility shift assays were done with each of the wild-type and mutant proteins incubated with RNA encompassing the target binding sequence within the $\mathrm{HO} 3^{\prime}$ UTR (Fig. 6A). As shown in Figure 6B, the wild-type Puf5RDp bound the $\mathrm{HO}$ target sequence (lane 3 ), while the wild-type Puf3RDp bound the HO RNA less well (lane 4) and GST protein alone did not bind (lane 2). Evaluation of complex weaker, with a $K_{\text {d,app }}$ of $1.25 \pm 0.11 \mu \mathrm{M}$ (Fig. 7A,B). The R1, $\mathrm{R} 3$, and R5 single point mutations all promoted a small increase in affinity toward the $H O$ RNA versus the wild-type Puf3RDp, with $K_{\mathrm{d} \text {,app }}$ values of $0.86 \pm 0.04 \mu \mathrm{M}, 0.85 \pm 0.04 \mu \mathrm{M}$, and $0.87 \pm 0.04 \mu \mathrm{M}$, respectively (Fig. 7A,B). Any combination of two point mutations (R1/R3, R1/R5, or R3/R5) promoted a slightly larger increase in affinity of the protein to the HO RNA, with $K_{\mathrm{d}, \text { app }}$ values of $0.74 \pm 0.03 \mu \mathrm{M}, 0.76 \pm 0.03 \mu \mathrm{M}$, and $0.77 \pm 0.03 \mu \mathrm{M}$, respectively (Fig. $7 \mathrm{~A}, \mathrm{~B}$ ). Since all combinations of double mutants promoted a similar enhanced affinity toward the $H O$ RNA, these results indicate that the three amino acid positions tested play equal roles in RNA binding, and the small contribution of each toward binding affinity is additive. This observation was verified by analysis of 
(A)

(B)

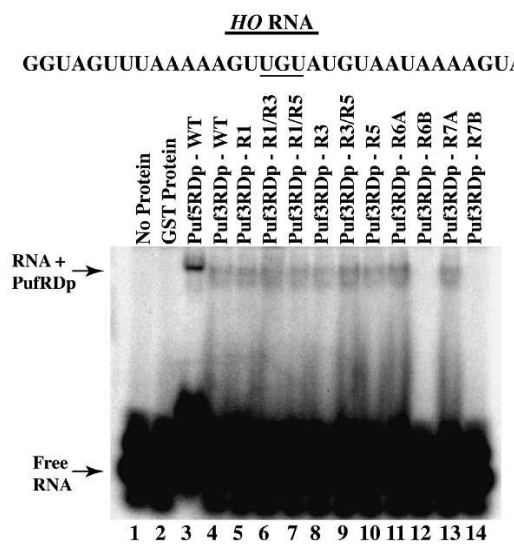

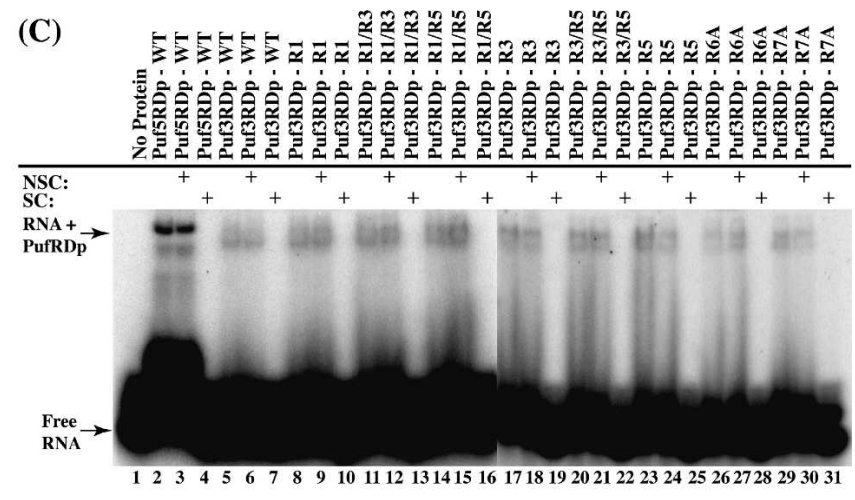

FIGURE 6. In vitro binding of wild-type and mutant Puf3RD proteins to HO RNA. (A) Sequence of the 35-nt $H O$ RNA transcript used in binding reactions is shown. The UGU core binding element is underlined. $(B, C)$ In vitro binding reactions of radiolabeled $H O$ RNA in the absence or presence of $0.65 \mu \mathrm{M}$ protein were separated on native polyacrylamide gels. Positions of unbound RNA (Free RNA) as well as RNA-Puf5RDp or RNA-Puf3RDp complexes (RNA+PufRDp) are indicated. (B) Reactions were performed in the presence of GST alone (lane 2), wild-type Puf5RDp (lane 3), wild-type Puf3RDp (lane 4), or mutant Puf3RD proteins (lanes 5-14). (C) The specificity of RNA-protein interactions was analyzed by using excess unlabeled vector RNA or HO RNA as nonspecific (NSC) and specific competitors (SC), respectively, in binding reactions with wild-type Puf5RDp (lanes 2-4), wild-type Puf3RDp (lanes 5-7), or mutant Puf3RD proteins (lanes 8-31). The presence of either competitor is marked $(+)$.

a triple mutant (R1/R3/R5), which showed an even greater increase in affinity to the $H O$ RNA, with a $K_{\mathrm{d} \text {,app }}$ of $0.65 \pm 0.02$. However, this affinity was still over twofold weaker than that of the wild-type Puf5RDp.

\section{DISCUSSION}

The conserved repeat domain of Puf proteins serves as the RNA binding surface as well as the protein docking point for interactions required for regulation of mRNA translation and degradation. We have characterized the interactions involved in RNA target specificity and mRNA decay regulation by the yeast Puf3 protein. First, we provided evidence that a single serine to cysteine point mutation in the first repeat (R1) inhibited binding and regulation of the COX17 mRNA by Puf3RDp. In vitro gel mobility shift assays showed no detectable binding of this R1 mutant to a target binding site in the COX17 $3^{\prime}$-UTR (Fig. 2), and
(A)

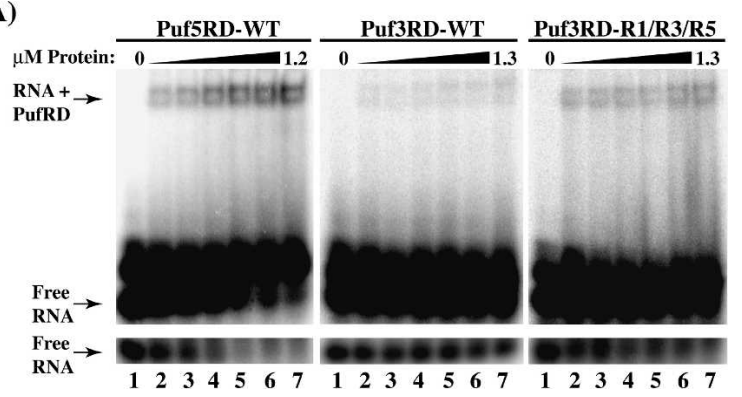

(B)

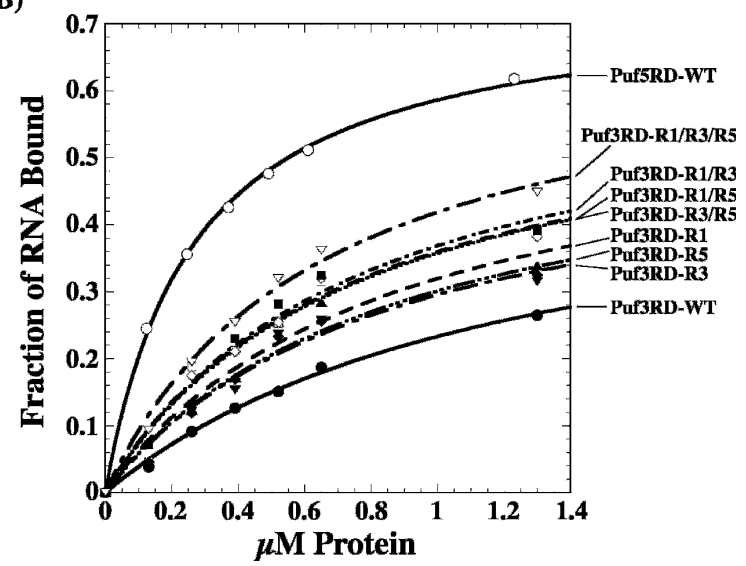

FIGURE 7. Comparison of binding affinities of wild-type and mutant Puf3RD proteins to HO RNA in vitro. (A) Gel mobility shift assays of in vitro binding reactions with radiolabeled HO RNA in the absence or presence of increasing concentrations of Puf5RDp-WT, Puf3RDp-WT, and Puf3RDp-R1/R3 are shown as representatives of similar assays performed on all mutant proteins. Concentrations of protein used in the Puf5RDpWT binding reactions were $0,0.12,0.24,0.37,0.49,0.61$, and $1.2 \mu \mathrm{M}$ in lanes $1-7$, respectively. Concentrations of protein used in all Puf3RDp binding reactions were $0,0.13,0.26,0.39,0.52,0.65$, and $1.3 \mu \mathrm{M}$ in lanes $1-7$, respectively. Positions of unbound RNA (Free RNA) as well as RNAPuf5RDp and RNA-Puf3RDp complexes (RNA+PufRDp) are indicated. Panels below each gel reflect a lighter exposure of the Free RNA within each respective gel. (B) Data from the gel mobility shift assays performed with Puf5RDp-WT, Puf3RDp-WT, and all Puf3RD inner surface point mutant proteins are plotted with the micromolar concentration of protein on the $X$-axis and the fraction of RNA shifted from free RNA to bound RNA on the $Y$-axis. Binding curves are shown for Puf3RDp-WT (filled circle), Puf5RDp-WT (open circle), Puf3RDp-R1 (filled triangle), Puf3RDp-R3 (filled inverted triangle), Puf3RDp-R5 (filled diamond), Puf3RDp-R1/R3 (open square), Puf3RDp-R1/R5 (open diamond), Puf3RDp-R3/R5 (filled square), and Puf3RDp-R1/R3/R5 (open inverted triangle). Data points are averages of multiple experiments. 
expression of the R1 mutant in a puf $3 \Delta$ strain failed to rescue rapid decay of the COX17 mRNA (Fig. 4), with deadenylation and decapping slowed to the same extent as in a puf $3 \Delta$ strain (Fig. 5). In contrast, neither a cysteine-to-threonine point mutation in repeat 3 nor an arginine-to-cysteine point mutation in repeat 5 had any detrimental effects on binding or regulation of COX17 mRNA. Both the R3 and R5 mutants showed wild-type binding affinities in vitro (Fig. 3), and both rescued wild-type decay of COX17 mRNA (Fig. 4) through rapid deadenylation and decapping (Fig. 5).

The results with the inner surface point mutations of the Puf3p repeat domain are surprising, given the predicted roles of these amino acids in binding to a target RNA. Based on the alignment with the HsPum crystal structure (Wang et al. 2002), the serine in the first repeat of Puf3p was predicted to make a specific van der Waals interaction with the final adenine of the COX17 target sequence (Fig. 1C). In Puf5p, a cysteine located at this position was predicted to make a similar van der Waals interaction with a final adenine of the HO RNA target sequence (Fig. 1C). Therefore, the serine-to-cysteine mutation in R1 of Puf3RDp was not expected to cause a significant change in the ability of Puf3RDp to bind the COX17 target RNA. In fact, Puf3p utilizes a cysteine in Repeat 3 to make a predicted van der Waals interaction with another adenine in the COX17 target sequence (Fig. 1C). However, since the serine to cysteine mutation in R1 abolished detectable RNA binding, this result demonstrates that the role of the $\mathrm{R} 1$ serine cannot be to simply make a low-energy van der Waals interaction with the adenine. It is actually quite remarkable that a single amino acid change would have such a drastic effect on a protein that utilizes 24 predicted RNA contacts over its extended eight-repeat structure. We do not believe that the cysteine introduction caused a reduction of protein activity due to disulfide bond formation, as RNA-protein binding buffer is made fresh with dithiothreitol (DTT) for each experiment. Moreover, the serine to cysteine mutation did not interrupt binding to the $\mathrm{HO}$ target sequence (Fig. 7), demonstrating that the R1 mutant protein is still active and thus likely folded correctly. Therefore, the R1 serine of Puf3p may be critical for intraprotein interactions, such as with other side-chains, to promote the precise architecture of the chemical surface to bind the COX17 RNA target. Alternatively, the R1 serine may be contacting the RNA downstream of the 8-nt core binding element, where there are several differences between the Puf3 and Puf5 target sites. Indeed, analysis of the FBF target binding site revealed that specific sequences both $5^{\prime}$ and $3^{\prime}$ of the core binding element are required for optimal binding, and it is hypothesized that these nucleotides may make novel contacts with the Puf protein (Bernstein et al. 2005).

Alignment with the HsPum crystal structure (Wang et al. 2002) places the cysteine of the Puf3p Repeat 3 in a van der Waals contact with an adenine (Fig. 1C), while Puf5p utilizes a threonine at this position to contact a guanine (Fig. 1C). Since the adenine versus guanine contact is the only difference between the Puf3p and Puf5p RNA target sequences used in these studies, we had originally predicted this amino acid position to be a point of target specificity. Previous work with the HsPum supported a modular role of each repeat, such that specificity is determined by three amino acids located at predicted RNA-binding positions of each repeat. In particular, directed alteration of the three RNA-binding amino acids within repeat 6 of the HsPum could alter target specificity (Wang et al. 2002). However, our results show that binding specificity is not necessarily modular. The finding that a cysteine-to-threonine mutation in R3 of Puf3RDp did not inhibit binding to the COX17 target RNA (Fig. 3) implies that other amino acids outside the three predicted positions of this repeat are promoting RNA binding specificity. This result also supports our hypothesis that RNA target specificity may be dependent on changes in protein surface architecture that are promoted by intraprotein interactions between amino acids that are not necessarily critical for direct contact with the RNA. Precedence for nucleic acid binding proteins that appear to act in a modular fashion but are, in fact, much more complex are the zinc-finger proteins. These proteins, similar to the Puf proteins, were originally thought to attain specificity to target sequences by the identity of amino acids at particular base-interacting positions within each zincfinger module. However, closer inspection has shown that intraprotein interactions such as side-chain-side-chain contacts and interactions with ordered water molecules are critical for determining binding specificity (Miller and Pabo 2001). We believe this might be occurring in the Puf proteins as well.

In contrast to the COX17 binding studies, analyses of the Puf3RDp mutants binding the HO RNA show that the identities of the amino acids at all three mutagenized RNA-interacting positions are important for binding to this Puf5p target RNA. In addition, amino acids outside these predicted positions are also critical for promoting binding affinity. Unlike the inability of Puf5p to bind the COX17 RNA, Puf3p weakly bound the HO RNA (Fig. 7), suggesting that the architecture of the Puf3p binding surface is flexible enough to accommodate binding to this RNA. Single mutations that replaced a Puf3p amino acid for that found in Puf5p promoted a small increase in affinity to the $H O$ RNA, while combinations of double mutations and a triple mutant promoted successively larger increases in affinity (Fig. 7). This supports a model in which each $H O$ RNA-protein contact makes a small but equal contribution to the binding energy, with the identity of the amino acid important for proper binding. However, while the Puf3RDp triple mutant effectively mimics 24 out of 24 predicted RNA-interacting amino acids of Puf5p, the affinity of this protein was still over twofold less than that of wild-type Puf5RDp toward the $\mathrm{HO}$ target 
(Fig. 7). This suggests that there are other amino acids unique to Puf5RDp that play significant roles in RNA binding. These amino acids may be involved in critical intraprotein interactions, or they may be contacting the RNA outside the 8-nt core binding element.

Key information was also obtained regarding Puf3p interactions required for mRNA decay regulation. The R7A deletion of amino acids RDKN, which are located at the beginning of a predicted 16-amino-acid loop structure on the protein's outer surface between repeats 7 and 8, abolished COX17 decay regulation (Fig. 4), with deadenylation and decapping slowed to the same extent as in a $p u f 3 \Delta$ strain (Fig. 5), while having no effect on binding of the protein to the mRNA (Fig. 3). It is likely that one or more of these amino acids are involved in protein-protein interactions that provide a signal to the decay machinery to rapidly degrade the bound mRNA. This signal could be involved in recruiting the decay machinery to the mRNA, or the signal could enhance the activity of the decay machinery, perhaps by altering the mRNP structure. The fact that the RDKN deletion disrupts both rapid deadenylation and rapid decapping suggests that regulation of these processes is linked by protein interactions at this site. Such interactions could involve direct contacts with regulators or components of both the deadenylation and decapping machineries, or these interactions could simply be altering the mRNP structure to allow more rapid access of the mRNA to both decay machineries. A corresponding loop region between repeats 7 and 8 of DmPum is required for protein interactions with Nanos and Brat, which are necessary for hunchback mRNA repression (Edwards et al. 2001). However, it is unknown how these interactions repress hunchback mRNA. While this site of protein interaction appears to be conserved between Puf proteins, it is intriguing that the characteristics of the loops in DmPum and Puf3p are quite different, with no conservation of sequence and the Puf3p loop being 11 amino acids longer. Efforts are underway to study the role of this loop in potential Puf3p protein interactions.

In contrast to the R7A deletion, the R6A deletion of amino acids FTNKEM, which our results suggest are located in an outer surface loop of Puf3p within repeat 6, had no deleterious effects on either binding (Fig. 3) or COX17 decay regulation (Fig. 4), with deadenylation and decapping occurring as rapidly as with wild-type Puf3RDp (Fig. 5). While these results show that this amino acid region plays no role in the decay of COX17 mRNA, it is possible that this region is important for regulation of other Puf3p target mRNAs. Precedence for a single Puf protein recruiting different sets of protein partners on different mRNA targets comes from Drosophila, where DmPum recruits both Nanos and Brat when bound to hunchback mRNA but only recruits Nanos when bound to cyclinB mRNA (Sonada and Wharton 2001). Future work will determine what roles the R7A and R6A loop domains play in decay regulation of other Puf3p target mRNAs.

\section{MATERIALS AND METHODS}

\section{Yeast strains}

The genotypes of all $S$. cerevisiae strains used in the study are as follows: yWO43 MAT $\alpha$, his4-539, leu2-3,112, trp1-1, ura3-52, cup1::LEU2/PM, rpb1-1, puf3::Neo ${ }^{r}$ (yRP1360) (Olivas and Parker 2000); yWO51 MATa, his4-539, leu2-3,112, trp1-1, ura3, rpb11, cox17::TRP1, puf3::Neo ${ }^{r}$ (yRP1547) (Olivas and Parker 2000).

\section{Plasmids}

The GST-PUF3 Repeat Domain (amino acids 465-879) fusion construct ( $\mathrm{pWO} 12$ ) was created in a derivative of pGEX-3X (Amersham Biosciences) as previously described (Jackson et al. 2004). To create pWO14, the PUF3 Repeat Domain (PUF3RD) was isolated from pWO12 and inserted into a derivative of pG-1 (Schena et al. 1991), placing the PUF3RD just downstream from an inserted Flag tag sequence and the GPD promoter as previously described (Jackson et al. 2004). Mutant PUF3RD constructs pWO29-pWO38 (Table 1) were created from pWO14, while pWO76 was created from pWO12 by using the QuikChange XL Site-Directed Mutagenesis Kit (Stratagene). All mutations were verified by sequencing. Mutant GST-PUF3RD fusion constructs pWO39-pWO47 and pWO75 (Table 1) were created by BamHI and SalI digestion of pWO29-pWO38 and insertion into a derivative of pGEX-3X using the same restriction sites. The pWO18 GST-PUF5RD fusion construct was created in pGEX-6p-3 (Amersham Biosciences) as previously described (Jackson et al. 2004).

\section{Protein expression and purification}

All GST fusion constructs were transformed into the protease deficient Escherichia coli strain BL-21, and GST fusion proteins were purified as recommended by the manufacturer. Protein eluates were dialyzed into $50 \mathrm{mM}$ Tris- $\mathrm{HCl}$ ( $\mathrm{pH} 8.0$ ), and expression products were verified by Western analysis with anti-GST antibodies.

\section{In vitro binding analyses}

Short COX17 Site-A and HO RNAs (29-30 nt) were transcribed from single-stranded oligonucleotide templates containing the 18-nt T7 RNA polymerase promoter annealed to a complementary primer. The T7-Megashortscript Kit (Ambion) was used to transcribe the RNAs with the following changes: The reactions contained $500 \mu \mathrm{M}$ each of ATP, CTP, and GTP; $50 \mu \mathrm{M}$ of UTP; $40 \mu \mathrm{Ci}$ of $\alpha-{ }^{32} \mathrm{P}$ UTP ( $800 \mathrm{Ci} / \mathrm{mmol}$ ) for labeled reactions; and $20 \mathrm{U}$ RNasin. Radiolabeled transcripts were purified via separation on denaturing polyacrylamide gels, elution from gel slices, and ethanol precipitation. Unlabeled transcripts were purified by using the Nucleotide Removal Kit (Qiagen).

Each $20 \mu \mathrm{L}$ RNA-protein binding reaction contained radiolabeled RNA $(20,000 \mathrm{cpm}, \sim 200 \mathrm{pM})$ and $1 \times$ binding buffer $(10$ mM HEPES at pH 7.5, $50 \mathrm{mM} \mathrm{KCl,} 1$ mM EDTA, 2 mM DTT, 200 $\mathrm{U} / \mathrm{mL}$ RNasin, $0.1 \mathrm{mg} / \mathrm{mL}$ bovine serum albumin, $0.01 \%$ Tween$20,0.1 \mathrm{mg} / \mathrm{mL}$ poly $(\mathrm{rU}), 10 \mu \mathrm{g} / \mathrm{mL}$ yeast tRNA) in the presence or absence of wild-type GST-Puf3RDp, mutant GST-Puf3RDp, or wild-type GST-Puf5RDp, and in the presence or absence of $\sim 10$ fold excess unlabeled transcript. Reactions were incubated at $24^{\circ} \mathrm{C}$ 
TABLE 1. Puf protein mutations and respective expression plasmids used in this study

\begin{tabular}{lllc}
\hline Protein & \multicolumn{1}{c}{ Mutation } & GST-plasmid & Yeast plasmid \\
\hline Puf3RDp & Wild type & pWO 12 & pWO 14 \\
Puf5RDp & Wild type & pWO 18 & - \\
Puf3RDp-R1 & S553C & pWO 39 & pWO 29 \\
Puf3RDp-R1/R3 & S553C and C625T & pWO 40 & pWO 30 \\
Puf3RDp-R1/R5 & S553C and R698C & pWO 41 & pWO 31 \\
Puf3RDp-R3 & C625T & pWO 42 & pWO 32 \\
Puf3RDp-R3/R5 & C625T and R698C & pWO 43 & pWO 33 \\
Puf5RDp-R5 & R698C & pWO 44 & pWO 34 \\
Puf3RDp-R6A & $\Delta$ F758-M763 & pWO 45 & pWO 35 \\
Puf3RDp-R6B & $\Delta$ I771-D776 & pWO 46 & pWO 36 \\
Puf3RDp-R7A & $\Delta$ R800-N803 & pWO 47 & pWO 37 \\
Puf3RDp-R7B & $\Delta$ N807-S812 & pWO 75 & pWO 38 \\
Puf3RDp-R1/R3/R5 & S553C, C625T, and R698C & pWO 76 & - \\
\hline
\end{tabular}

for $30 \mathrm{~min}, 5 \mu \mathrm{g}$ of heparin was added, and then reactions incubated a further $10 \mathrm{~min}$ at $24^{\circ} \mathrm{C}$. Reactions were electrophoresed on $8 \%$ nondenaturing polyacrylamide gels at $200 \mathrm{~V}$ for $2.5 \mathrm{~h}$ at $4{ }^{\circ} \mathrm{C}$. Apparent $K_{\mathrm{d}}$ values were determined by first calculating the fraction of RNA bound for each gel lane using the following definition: Fraction Bound $=$ Shifted RNA/(Shifted RNA + Free RNA), where Shifted RNA and Free RNA represent the storage phosphor signal of all shifted complexes or the free RNA species, respectively. Binding-incompetent RNAs appeared in some RNA preparations and had a more retarded mobility than did the expected free RNA. Since the amount of these RNAs remained fairly constant within an experiment and did not reflect changes in protein concentrations, these RNAs were likely a product of structural changes in the radiolabeled RNA and were not included in the calculations. KaleidaGraph 3.5 software was used to fit the binding data to the Langmuir isotherm: Fraction of RNA Bound $=[$ Protein $] /\left(K_{\mathrm{d} \text {,app }}+[\right.$ Protein $\left.]\right)$. The confidence levels for all binding fits with the COX17 RNA were $>99 \%$, and confidence levels were $>98 \%$ for all binding fits with the HO RNA. Error was determined as the standard deviation of the average $K_{\mathrm{d} \text {,app }}$ from multiple experiments.

\section{In vivo decay analysis}

Steady-state transcriptional shut-off experiments were performed essentially as described (Caponigro et al. 1993) on yWO43 ( $p u f 3 \Delta$ ), which contains the rbp1-1 temperature-sensitive allele for RNA polymerase II. yWO43 was also analyzed after transformation with plasmids expressing the wild-type Puf3RDp (pWO14) as well as the mutant Puf3RDp's (pWO29-38). Northern blots were normalized for loading by using the stable RNA polymerase III transcript, scRI RNA (Felici et al. 1989).

Transcriptional pulse-chase experiments were performed essentially as described (Decker and Parker 1993) on yWO51 (cox17A, puf $3 \Delta, r b p 1-1)$. Regulated expression of COX17 RNA was accomplished by transformation of yWO51 with pWO5 (pG74/ST30) (Beers et al. 1997), in which the COX17 gene is under the control of the GAL10 promoter. In addition, yWO51 was transformed with pWO14 (pPuf3RD-WT) or pWO29-38 (pPuf3RD-mutants). Transcription was induced for $8 \mathrm{~min}$ by the addition of galactose and then rapidly repressed by simultaneously adding glucose and shifting the culture to $37^{\circ} \mathrm{C}$. Poly(A) tail lengths were monitored by the cleavage of COX17 mRNA just upstream of the stop codon using $\mathrm{RNaseH}$ reactions with oWO1 as described (oCOX17-C) (Olivas and Parker 2000). RNA was separated on $6 \%$ denaturing polyacrylamide gels at $300 \mathrm{~V}$ for $4 \mathrm{~h}$ and then transferred to nylon membrane for probing with radiolabeled oWO2 (oCOX17-P, Olivas and Parker 2000).

\section{ACKNOWLEDGMENTS}

We gratefully thank Cynthia Dupureur, Elizabeth Kellogg, Theresa Thiel, and members of the Olivas laboratory for helpful discussions and review of the manuscript. This work was supported by a grant to W.M.O. from the National Institutes of Health (GM63759).

Received July 20, 2005; accepted August 10, 2005.

\section{REFERENCES}

Beers, J., Glerum, D.M., and Tzagoloff, A. 1997. Purification, characterization and localization of yeast COX17p, a mitochondrial copper shuttle. J. Biol. Chem. 272: 33191-33196.

Bernstein, D., Hook, B., Hajarnavis, A., Opperman, L., and Wickens, M. 2005. Binding specificity and mRNA targets of a C. elegans PUF protein, FBF-1. RNA 11: 447-458.

Caponigro, G. and Parker R. 1996. Mechanisms and control of mRNA turnover in Saccharomyces cerevisiae. Microbiol. Rev. 60: 233-249.

Caponigro, G., Muhlrad, D., and Parker, R. 1993. A small segment of the MATal transcript promotes mRNA decay in Saccharomyces cerevisiae: A stimulatory role for rare codons. Mol. Cell. Biol. 13: 5141-5148.

Decker, C.J. and Parker, R. 1993. A turnover pathway for both stable and unstable mRNAs in yeast: evidence for a requirement for deadenylation. Genes \& Dev. 7: 1632-1643.

Derrigo, M., Cestelli, A., Savettieri, G., and Di Liegro, I. 2000. RNAprotein interactions in the control of stability and localization of messenger RNA. Int. J. Mol. Med. 5: 111-123.

Edwards, T.A., Pyle, S.E., Wharton, R.P., and Aggarwal, A.K. 2001. Structure of Pumilio reveals similarity between RNA and peptide binding motifs. Cell 105: 281-289.

Felici, F., Cesareni, G., and Hughes, J.M.X. 1989. The most abundant small cytoplasmic RNA of Saccharomyces cerevisiae has an important function required for normal cell growth. Mol. Cell. Biol. 9: 3260-3268.

Gerber, A.P., Herschlag, D., and Brown, P.O. 2004. Extensive association of functionally and cytotopically related mRNAs with Puf family RNA-binding proteins in yeast. PLoS. Biol. 2: 342-354.

Gray, N.K. and Wickens, M. 1998. Control of translation initiation in animals. Annu. Rev. Cell Dev. Biol. 14: 399-458.

Grzybowska, E.A., Wilczynska, A., and Siedlecki, J.A. 2001. Regulatory functions of $3^{\prime}$ UTRs. Biochem. Biophys. Res. Commun. 288: 291295.

Gu, W., Deng, Y., Zenklusen, D., and Singer, R.H. 2004. A new yeast PUF family protein, Puf6p, represses ASH1 mRNA translation and is required for its localization. Genes \& Dev. 18: 1452-1465.

Guex, N. and Peitsch, M.C. 1997. SWISS-MODEL and the SwissPdbViewer: An environment for comparative protein modeling. Electrophoresis 18: 2714-2723. 
Guhaniyogi, J. and Brewer, G. 2001. Regulation of mRNA stability in mammalian cells. Gene 265: 11-23.

Jackson, J.S., Houshmandi, S.S., Leban, F.L., and Olivas W.M. 2004. Recruitment of the Puf3 protein to its mRNA target for regulation of mRNA decay in yeast. RNA 10: 1625-1636.

Kraemer, B., Crittenden, S., Gallegos, M., Moulder, G., Barstead, R., Kimble, J., and Wickens, M. 1999. NANOS-3 and FBF proteins physically interact to control the sperm-oocyte switch in Caenorhabditis elegans. Curr. Biol. 9: 1009-1018.

Luitjens, C., Gallegos. M., Kraemer, B., Kimble, J., and Wickens, M. 2000. CPEB proteins control two key steps in spermatogenesis in C. elegans. Genes \& Dev. 14: 2596-2609.

Mazumder, B., Seshadri, V., and Fox, P.L. 2003. Translational control by the $3^{\prime}$-UTR: The ends specify the means. Trends Biochem. Sci. 28: 91-98.

Miller, C.J. and Pabo, C.O. 2001. Rearrangement of side-chains in a Zif268 mutant highlights the complexities of zinc finger-DNA recognition. J. Mol. Biol. 313: 309-315.

Murata, Y. and Wharton, R. 1995. Binding of Pumilio to maternal hunchback mRNA is required for posterior patterning in Drosophila embryos. Cell 80: 747-756.

Nakahata, S., Katsu, Y., Mita, K., Inoue, K., Nagahama, Y., and Yamashita, M. 2001. Biochemical identification of Xenopus Pumilio as a sequence-specific cyclin B1 mRNA-binding protein that physically interacts with a Nanos homolog, Xcat-2, and a cytoplasmic polyadenylation element-binding protein. J. Biol. Chem. 276: 20945-20953.

Olivas, W. and Parker, R. 2000. The Puf3 protein is a transcript-specific regulator of mRNA degradation in yeast. EMBO J. 19: 6602-6611.

Peitsch, M.C. 1995. Protein modeling by e-mail. Bio/Technology 13: 658-660.

Schena, M., Picard, D., and Yamamoto, K.R. 1991. Vectors for constitutive and inducible gene expression in yeast. Methods Enzymol. 194: $389-398$.
Schwede, T., Kopp, J., Guex, N., and Peitsch, M.C. 2003. SWISSMODEL: An automated protein homology-modeling server. Nucleic Acids Res. 31: 3381-3385.

Sonada, J. and Wharton, R.P. 1999. Recruitment of Nanos to hunchback mRNA by Pumilio. Genes \& Dev. 13: 2704-2712.

. 2001. Drosophila Brain Tumor is a translational repressor. Genes \& Dev. 15: 762-773.

Tadauchi, T., Matsumoto, K., Herskowitz, I., and Irie, K. 2001. Post-transcriptional regulation through the HO $3^{\prime}$-UTR by Mpt5, a yeast homolog of Pumilio and FBF. EMBO J. 20: 552-561.

Wang, X., Zamore, P.D., and Tanaka Hall, T.M. 2001. Crystal structure of a Pumilio homology domain. Mol. Cell 7: 855-865.

Wang, X., McLachlan, J., Zamore, P.D., and Tanaka Hall, T.M. 2002. Modular recognition of RNA by a human Pumilio-homology domain. Cell 110: 501-512.

Wharton, R.P., Sonoda, J., Lee, T., Patterson, M., and Murata, Y. 1998. The Pumilio RNA-binding domain is also a translational regulator. Mol. Cell 1: 863-872.

White, E.K., Moore-Jarrett, T., and Ruley, H. E. 2001. PUM2, a novel murine Puf protein, and its consensus RNA-binding site. RNA 7: $1855-1866$.

Wickens, M., Bernstein, D.S., Kimble, J., and Parker, R. 2002. A PUF family portrait: $3^{\prime} \mathrm{UTR}$ regulation as a way of life. Trends Genet. 18: 150-157.

Zamore, P.D., Williamson, J.R., and Lehmann, R. 1997. The pumilio protein binds RNA through a conserved domain that defines a new class of RNA-binding proteins. RNA 3: 1421-1433.

Zamore, P.D., Bartel, D.P., Lehmann, R., and Williamson, J.R. 1999. The PUMILIO-RNA interaction: A single RNA-binding domain monomer recognizes a bipartite target sequence. Biochemistry 38: 596-604.

Zhang, B., Gallegos, M., Puoti, A., Durkin, E., Fields, S., Kimble, J., and Wickens, M.P. 1997. A conserved RNA-binding protein that regulates sexual fates in the $C$. elegans hermaphrodite germ line. Nature 390: 477-484. 

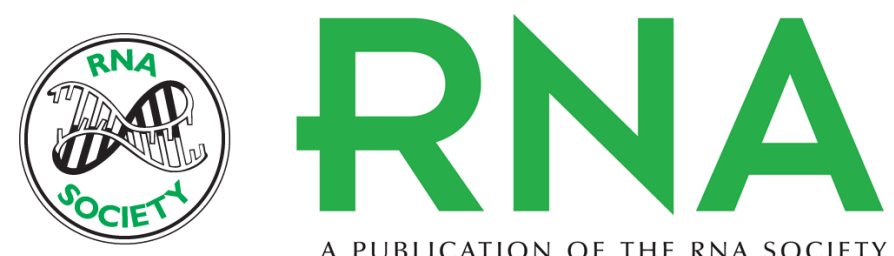

A PUBLICATION OF THE RNA SOCIETY

\section{Yeast Puf3 mutants reveal the complexity of Puf-RNA binding and identify a loop required for regulation of mRNA decay}

S. SEAN HOUSHMANDI and WENDY M. OLIVAS

RNA 2005 11: 1655-1666

References This article cites 35 articles, 16 of which can be accessed free at: http://rnajournal.cshlp.org/content/11/11/1655.full.html\#ref-list-1

License

Email Alerting Receive free email alerts when new articles cite this article - sign up in the box at the top Service right corner of the article or click here. 